\title{
Gossypiboma Masquerading as Small Bowel Malignancy
}

CrossMark

\author{
Amir H Boghratian 1,*, Ahmad M Al-Taee ${ }^{2}$
}

\section{ABSTRACT}

1. Division of Gastroenterology, Iran University of Medical Sciences, Tehran, Iran

2. Division of Gastroenterology and Hepatology, Saint Louis University, St Louis, Missouri, USA

\section{* Corresponding Author:}

Amir H Boghratian, MD Iran Mehr Hospital, Dorahi-e-Gholhak., Shariati Ave., Tehran, Iran Tel: + 982122009071 Fax: + 982122602649 Email: boghratianmd@yahoo.com

Received: 29 Nov. 2019 Accepted: 19 Feb 2020
Gossypiboma refers to inadvertently retained foreign objects after surgical operations. High body mass index and emergency surgery are risk factors of the condition. Presenting symptoms are usually non-specific and it may result in serious complications such as bowel obstruction. Removal can be attempted with endoscopic or surgical approaches depending on the site and presence of complications. This case highlights the need for considering gossypiboma in patients presenting with abdominal symptoms after recent abdominal surgery. Herein we report the case of a 30-year-old woman with abdominal pain two months after mini-gastric bypass surgery. Cross-sectional imaging showed a hyperdense area in the small bowel concerning for malignancy. However, upper endoscopy revealed a $10 \times 40 \mathrm{~cm}$ retained surgical gauze in the afferent loop that was successfully retrieved. Although rare, retained foreign body should be considered in the differential diagnosis of postoperative abdominal pain.

\section{KEYWORDS:}

Gossypiboma, Bariatric surgery, Retained foreign body

Please cite this paper as:

Boghratian AH, Al-Taee AM.Gossypiboma Masquerading as Small Bowel Malignancy. Middle East J Dig Dis 2020;12:123-125. doi: 10.34172/mejdd.2020.172.

\section{INTRODUCTION}

Gossypiboma is the term used to describe surgical equipment accidentally retained inside the body of a patient after surgery. The term "gossypiboma" is derived from the Latin words gossypium ("cotton wool, cotton") and the suffix -oma, meaning a tumor or growth and illustrates a material within a patient's body consisting of a cotton matrix surrounded by a foreign body granuloma. It is a rare surgical complication but can result in significant morbidity and mortality. ${ }^{1}$

\section{CASE REPORT}

A 30-year-old woman presented to our clinic with a 3-week history of nausea, non-bloody vomiting, and abdominal pain. Medical history was notable for morbid obesity with a body mass index (BMI) of $42.8 \mathrm{~kg}$ / $\mathrm{m}^{2}$ Surgical history was notable for mini gastric bypass surgery, which was performed 2 months earlier. Vital signs were within normal limits. Abdominal examination revealed a distended abdomen with epigastric tenderness but without rebound tenderness or rigidity. Bowel sounds were diminished. Laboratory testing including a complete blood count, 


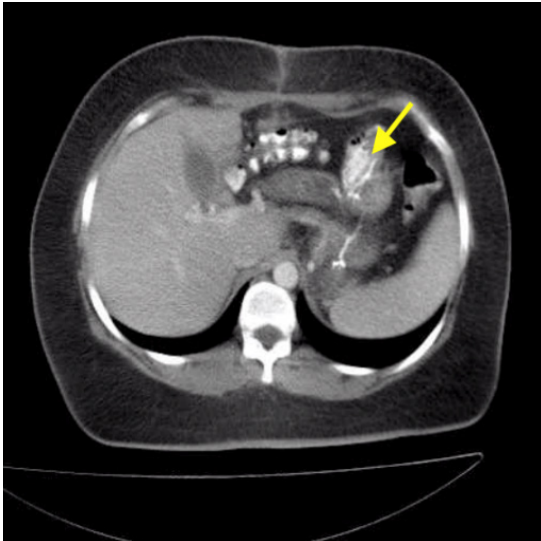

Fig.1: Computed tomography (CT) of the abdomen with intravenous contrast showed a hyperdense area in the small bowel

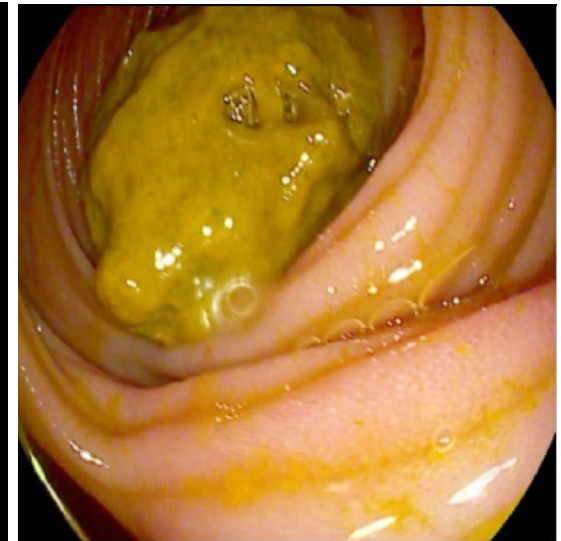

Fig.2: Upper endoscopy which showed a $3 \times 4 \mathrm{~cm}$ clean based ulcer proximal to a large greenish foreign body that obstructed the afferent loop

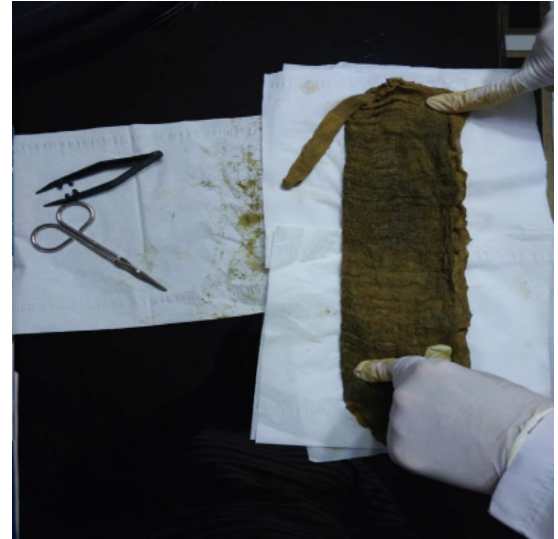

Fig.3: A biopsy forceps and was found to be a $10 \times 40 \mathrm{~cm}$ retained gauze covered with bilious exudates comprehensive metabolic panel, and urinalysis were within normal limits. She was seen by her physician on multiple occasions. Her symptoms did not improve over time.

Abdominal sonography was inconclusive. Computed tomography (CT) of the abdomen with intravenous contrast showed a hyperdense area in the small bowel concerning for malignancy (figure 1). The patient underwent an upper endoscopy, which showed a $3 \times 4 \mathrm{~cm}$ clean based ulcer proximal to a large greenish foreign body that obstructed the afferent loop (figure 2). This was successfully retrieved with a biopsy forceps and was found to be a $10 \times 40 \mathrm{~cm}$ retained gauze covered with bilious exudates (figure 3 ).

The patient's symptoms resolved after upper endoscopy and she was discharged on oral pantoprazole $40 \mathrm{mg}$ daily. Follow-up upper endoscopy 3 months later showed complete healing of the afferent wall ulcer.

\section{DISCUSSION}

Retained surgical sponge or gossypiboma is the most commonly retained foreign material in the body after surgical operations, which lead to serious damages. The condition is often underestimated because case numbers are calculated only on the basis of the fear of medicolegal repercussions. There are several case reports that presented gossypiboma retained in the various parts of the body including abdomen, ${ }^{2}$ pericardium,${ }^{3}$ chest,${ }^{4}$ right proximal leg, ${ }^{5}$ and shoulder, ${ }^{6}$ and it happened even after neurosurgical operations. ${ }^{7}$ In our case report, we presented for the first time a gossypiboma retained in the afferent loop after elective bariatric surgery, gastrojejunostomy.

Gawande and colleagues evaluated the risk factors for retained foreign material in the body during the operation. They showed that emergency surgery, unplanned change in the operation, and high BMI were significantly associated with a higher risk of retained foreign material in the body. Emergency surgery was associated with a 9-fold increased risk of retained material in the operation. ${ }^{8}$ It seems that in our case, excessive BMI had a more important role in increasing the risk of gossypiboma.

A history of previous surgery is a crucial clue for the diagnosis of gossypiboma at any part of the body. Recurrent abdominal pain, nausea, vomiting, and abdominal distention in a patient who had a recent abdominal operation should raise the suspicion for retained foreign material in the abdomen. ${ }^{9}$ Nevertheless, the symptoms are non-specific and may not be taken seriously for days or even years. ${ }^{10}$ Imaging can be helpful in detecting retained foreign bodies. The CT findings of gossypiboma are described as well circumscribed masses with a thick wall; with or without gas. It should be noted that some post-operative changes such as air bubbles, calcifications, and edema may lead to confusion. ${ }^{11,12}$

There are some approaches to avoid gossypiboma. Sponge count is one of the most widespread methods where surgical checklists are used seriously and routinely for every patient. Traditionally, sponges are counted twice 
manually by two people at the beginning and before closing the wound, and the surgeon carefully considers the use of small sponges in large cavities and performs a methodical wound examination. Besides, radiologically detectable sponges and towels can be used in the surgical site and intra-operative radiography to count the gauze can be applied. Another technology is tagged surgical sponge with an electronic article surveillance system, which can be used to trace gauze electronically. ${ }^{13}$ Barcode scan can be used for all sponges, and all would be detectable with barcode scanners. ${ }^{9}$ However, these new technologies are not yet widely available.

Gossypiboma refers to inadvertently retained foreign objects after surgical operations. High BMI and emergency surgery are risk factors. Presenting symptoms are usually non-specific and it may result in serious complications such as bowel obstruction. Removal can be attempted with endoscopic or surgical approaches depending on the site and presence of complications. Our presented case highlights the need for considering gossypiboma in patients presenting with abdominal symptoms after recent abdominal surgery. Although rare, retained foreign body should be considered in the differential diagnosis of postoperative abdominal pain.

\section{ACKNOWLEDGMENT}

We are gratefully thankful to the medical staff of the endoscopy unit at Iran Mehr Hospital. We would also like to thank Dr. Masoomeh Kazemian, Dr. Hossein Saeedimotahar, and professor Dr. Homayoon Shahidi for their excellent assistance and guidance.

\section{ETHICAL APPROVAL}

There is nothing to be declared.

\section{CONFLICT OF INTEREST}

The authors declare no conflict of interest related to this work.

\section{REFERENCES}

1. Gibbs VC, Coakley FD, Reines HD. Preventable errors in the operating room: retained foreign bodies after surgery-Part I. Curr Probl Surg 2007;44:281-337. doi: 10.1067/j.cpsurg.2007.03.002.

2. Lincourt AE, Harrell A, Cristiano J, Sechrist C, Kercher K, Heniford T. Retained foreign bodies after surgery. J Surg Res 2007;138:170-4. doi: 10.1016/j. jss.2006.08.001.

3. Coşkun M, Boyvat F, Ağildere AM. CT features of a pericardial gossypiboma. Eur Radiol 1999;9:728-30. doi: $10.1007 / \mathrm{s} 003300050743$.

4. Sheehan RE, Sheppard MN, Hansell DM. Retained intrathoracic surgical swab: CT appearances. $J$ Thorac Imaging 2000;15:61-4. doi: 10.1097/00005382200001000-00012.

5. Lo CP, Hsu CC, Chang TH. Gossypiboma of the leg: MR imaging characteristics. A case report. Korean $J$ Radiol 2003;4:191-3. doi: 10.3348/kjr.2003.4.3.191.

6. Bevernage C, Geusens E, Nijs S. Case report: a gossypiboma in the shoulder. Emerg Radiol 2006;12:231-3. doi: 10.1007/s10140-006-0480-1.

7. Mathew JM, Rajshekhar V, Chandy MJ. MRI features of neurosurgical gossypiboma: report of two cases. Neuroradiology 1996;38:468-9. doi: 10.1007/ bf00607280.

8. Gawande AA, Studdert DM, Orav EJ, Brennan TA, Zinner MJ. Risk factors for retained instruments and sponges after surgery. $N$ Engl J Med 2003;348:22935. doi: 10.1056/NEJMsa021721

9. Lata I, Kapoor D, Sahu S. Gossypiboma, a rare cause of acute abdomen: A case report and review of literature. Int J Crit Illn Inj Sci 2011;1:157-60. doi: 10.4103/22295151.84805 .

10. Apter S, Hertz M, Rubinstein ZJ, Zissin R. Gossypiboma in the early post-operative period: a diagnostic problem. Clin Radiol 1990;42:128-9. doi: 10.1016/ s0009-9260(05)82084-7.

11. Umunna J. Gossypiboma and its implications. $J$ West Afr Coll Surg 2012;2:95-105.

12. Malik A, Jagmohan P. Gossypiboma: US and CT appearance. Indian J Radiol Imaging 2002;12:503-4.

13. Fabian CE. Electronic tagging of surgical sponges to prevent their accidental retention. Surgery 2005;137:298301. doi: 10.1016/j.surg.2004.10.003. 